\title{
Cambio climático: efectos sobre el suelo y la agricultura
}

\author{
Recibido: 15 Febrero 2013 - Revisado: 30 Abril 2013
}

Aceptado: 30 Mayo 2013 - Publicado: 30 Junio 2013

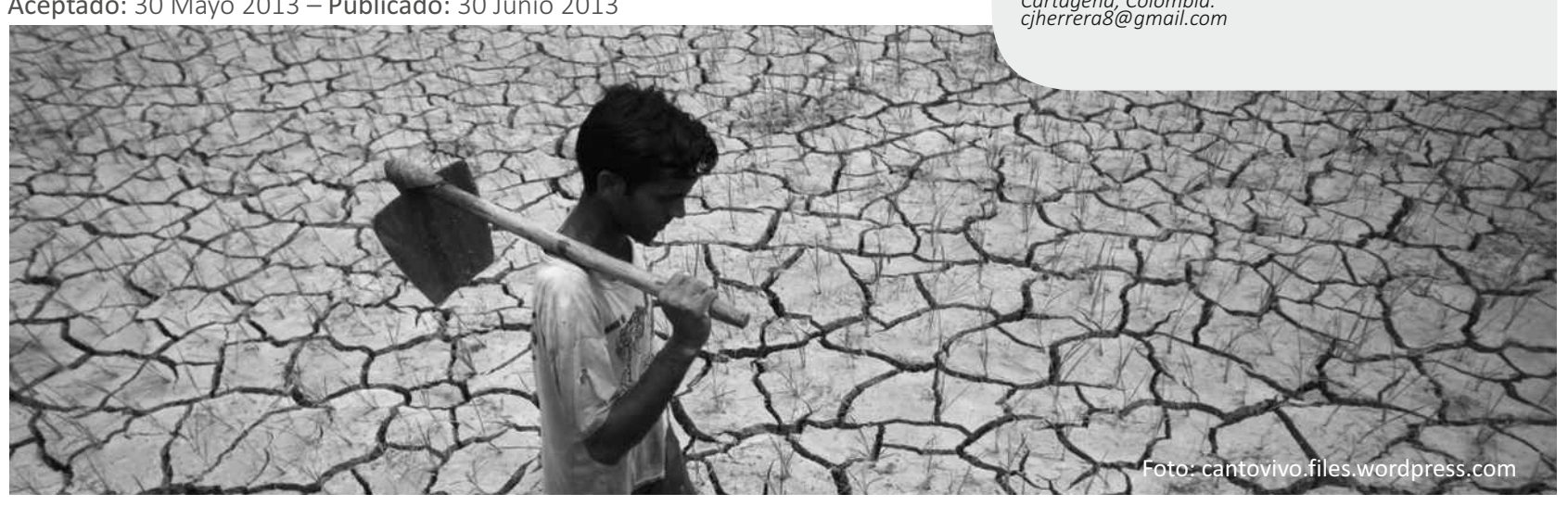

Resumen: En términos generales, el cambio climático se refiere a toda permutación de variabilidad significativa en las condiciones climáticas, que genera efectos a nivel: (a) ambiental, tales como alteraciones del sistema climático global (se destaca el aumento de la temperatura promedio global y las alteraciones en el régimen hidrológico) y el aumento en la ocurrencia de desastres naturales, y (b)socioeconómico en cuanto al aumento de la pobreza, la disminución de la productividad agrícola y el entorpecimiento del desarrollo económico. No obstante, a esta definición se le suman y/o restan elementos que generan una variedad de concepciones supeditadas al grado de aceptación del mismo por parte del ente o la persona que lo proponga.

El presente artículo presenta los distintos planteamientos acerca del cambio climático y describe la concepción acerca del tema en el ámbito mundial. Además, expone los retos de adaptación asumidos por comunidades y presenta propuestas generales para aquellas poblaciones que están sufriendo estragos a causa del cambio climático. Por último, llevado al contexto local, el artículo demuestra cómo un fenómeno natural o antrópico está impactando sobre la industria agropecuaria en Colombia. Para ello, se realizó una búsqueda detallada en diferentes bases de datos y artículos actualizados relacionados con la temática.

Palabras claves: Adaptación, cambio climático, gases de efecto invernadero.

Abstract: Climate change refers to any change in significant variability in climatic conditions, which generates effects at different levels: (a) environmental changes such as global climate system (chiefly the increase in global average temperatures and changes in the hydrological regime) and the increase in natural disasters; and (b) socio-economic in terms of increased poverty, declining agricultural productivity, and the hampering of economic development. However, some elements may beaded/subtracted to this definition, which produce a variety of conceptions that are contingent upon the degree of acceptance by either the entity or person who proposes it. This article presents both the different approaches on climate change and describes the conception about the issue at a global level. It also exposes the challenges of adaptation undertaken by communities and presents comprehensive proposals for populations that are being affected due to climate change. Lastly, the paper shows, within a local context, how a natural or man-made phenomenon is impacting the agricultural industry in Colombia. For this, a detailed search was conducted using different databases and updated articles related to the topic.

Key words: Adaptation, climate change, greenhouse effect gases 


\section{INTRODUCCIÓN}

La Convención Marco de las Naciones Unidas sobre Cambio Climático (CMNUCC) define el cambio climático como una transformación del clima atribuido directa o indirectamente a la actividad humana, que altera la composición de la atmósfera mundial y que se suma a la variabilidad natural de las condiciones climáticas observadas durante periodos de tiempo comparables. Por su parte, el Panel Intergubernamental de Expertos sobre Cambio Climático (IPCC) (IPCC, 2007) define el cambio climático como la variación estadísticamente significativa, ya sea de las condiciones climáticas medias o de su variabilidad, que se mantiene durante un periodo prolongado en el tiempo.

El cambio climático es considerado el problema ambiental más importante al que se ha enfrentado la humanidad hasta el presente (Velázquez, 2008). Los informes generados por el IPCC en 1990, 1995, 2001 y 2007 han permitido ampliar el análisis científico y la difusión de la problemática a la comunidad. Tales informes han generado un interés general, producto de las proyecciones caóticas y, en algunos casos, apocalípticas planteadas por diferentes estudios, algunos científicos y otros medios.

Para el IPCC (2007), el término cambio climático denota toda transformación del clima a lo largo del tiempo, independientemente de si es debido a la variabilidad natural o debido a la actividad humana. Además, expone que las principales causas de este problema ambiental son: (a) las emisiones de gases de efecto invernadero (GEI) de larga permanencia y (b) el forzamiento radiactivo del sistema climático, en donde predominan los GEI de larga permanencia y las emisiones mundiales de GEI causadas por actividades humanas (que han aumentado desde la era preindustrial en un $70 \%$ entre 1970 y 2004).

De la misma forma, el IPCC plantea que los principales precursores del cambio climático son: las variaciones experimentadas por las concentraciones de los GEI, los aerosoles en la atmósfera y la radiación solar, que altera el balance de energía del sistema climático. Asimismo, las concentraciones de $\mathrm{CO} 2, \mathrm{CH} 4$ y N2O en la atmósfera se han incrementado considerablemente por efecto de las actividades humanas desde 1750 y en la actualidad, exceden los valores preindustriales determinados mediante el análisis de núcleos de hielo acumulados durante miles de años.

Por otro lado, el Panel Internacional No Gubernamental Sobre Cambio Climático (NIPCC) tiene un planteamiento contrario al del IPCC. El proyecto del NIPCC fue concebido y dirigido por el Dr. S. Fred Singer, profesor emérito de Ciencias Ambientales en la Universidad de Virginia. EI NIPCC aseguran que aunque se esté pasando por un calentamiento, no hay indicios de que sus causas sean $100 \%$ antrópicas. Afirman, además, que debería de ser obvio que hechos como el derretimiento de glaciares y la desaparición de hielo ártico son enteramente irrelevantes para averiguar sobre las causas de este problema ambiental.

Cualquier calentamiento significativo, sea antrópico o natural, derretirá al hielo con frecuencia, muy lentamente. Por consiguiente, cualquier afirmación que exprese que está ocurriendo un calentamiento causado por el hombre está simplemente confundiendo las consecuencias del problema con sus causas, un común error de lógica. Además, las fluctuaciones de masa de los glaciares dependen de muchos factores además de la temperatura, tal como la cantidad de precipitación; por ello, los glaciares son una pobre forma de medir el calentamiento global (Fred, 2008).

Las investigaciones sobre el cambio climático no siguen la concepción de una investigación científica tradicional tras resultados definitivos; toman distancia de su eventual aplicación. Por el contrario, el descubrimiento del calentamiento global ha sido conducido por científicos dispersos que espontáneamente establecen contacto interdisciplinario, en un esfuerzo por entender la complejidad de los fenómenos del cambio climático. De esta forma los resultados nunca serán definitivos y siempre están orientados a su aplicación.

A pesar de que es un tema complejo, muchos políticos se han hecho los inconscientes de la situación; no obstante, los periodistas científicos, a través de los medios de comunicación masivos, han sido instrumentos poderosos para movilizar a la ciudadanía en la toma de medidas de mitigación y adaptación al cambio climático en su doble condición de consumidores y de ciudadanos.

Por ejemplo: en el caso del cloro-fluoro-carbono (CFC), han logrado sacarlos aerosoles del mercado en breve plazo. Con 
respecto a la lluvia ácida, se han aprobado políticas basadas en incentivos a la reducción de emisiones de $\mathrm{SO} 2$ al más bajo costo para los contribuyentes. Ambos casos han dado resultados exitosos, en buena medida, por haber sido elegidos por periodistas científicos en función de su fuerza noticiosa. Sucesos como estos movilizan a las poblaciones a exigir al poder político, estrategias para sus beneficios frente a las condiciones que mostrará el cambio climático impulsado por el calentamiento global.

\section{CONCEPCIÓN GLOBAL FRENTE AL CAMBIO CLIMÁTICO}

El calentamiento global es un tema concreto sobre el cual se debe establecer estrategias rápidas y sustentables para que no sea un problema que deje consecuencias a gran escala en el mundo. Las medidas que actúen sobre las causas del calentamiento recién empezarán a tener efectos en 30años más y dependen más de los países desarrollados que de aquellos llamados «en desarrollo». A estos últimos, en las próximas décadas, no nos queda mucho más que la mitigación de sus efectos y la adaptación (prepararnos para que no nos tomen desprevenidos).

Típicamente, son los más pobres quienes sufren más los efectos del cambio climático. Es más, algunos de los obstáculos a la adaptación y mitigación pueden ser atribuidos a las burocracias nacionales: (1) subestimación de las capacidades locales adquiridas en sus enfrentamientos a desastres naturales; (2) alto grado de centralización y sectorialización de políticas públicas que no dan cuenta de las diferencias entre localidades, lo que hace imposible medidas y políticas integrales de mitigación y adaptación; (3) ineficiencia en la administración y manejo de los recursos financieros y técnicos de las políticas públicas.

Esto indica que las estrategias o planes de mitigación y adaptación no se hallan muy claros en nuestra sociedad en desarrollo. En cuanto a la solidaridad entre naciones, no son los países en desarrollo los que deben ser apremiados para reducir sus emisiones de gases de efecto invernadero si con ello sacrifican su crecimiento económico, sino los países desarrollados.

La condición puesta por Brasil de ser compensado por ceder ante exigencias externas de detener la deforestación amazónica siempre surge en estos debates. Sin embargo, reconociendo la justicia de esta posición, no hubo ponencias, pero sí discusión y conversaciones sobre la aparición de mercados verdes, que han surgido espontáneamente a través de diferentes instrumentos. El de mayor desarrollo es el crédito en bonos por reducción de emisiones de $\mathrm{CO} 2$ vía eficiencia productiva (crecimiento) de empresas en países en desarrollo, que es pagado por sus pares de países desarrollados con dificultades de cumplir sus propias metas de reducción de emisiones. Finalmente, para afrontar el cambio climático cada nación debe afrontar la posición que debe, podría encontrar igualdad y mejores resultados para todos (Geisse, 2007).

La Convención de Naciones Unidas, aprobada en Nueva York el 9 de mayo de 1992, define el cambio climático como «un cambio de clima, atribuido directa o indirectamente a la actividad humana, que altera la composición de la atmósfera mundial y se suma a la variabilidad natural del clima observada durante periodos de tiempo comparables». Señala, además, que las emisiones de gases de efecto invernadero son uno de los principales causantes de este cambio.

Este planteamiento se viene manteniendo en los últimos 30 años, dando lugar a una extensa regulación de la Unión Europea y sus estados miembros, así como en el contexto internacional. En el ámbito mundial, las normas básicas son la propia Convención de Cambio Climático y el Protocolo de Kioto, cuya validez comprende el periodo del 1 de enero de 1998 a 31 de diciembre de 2012.

Mientras que en la Convención de Cambio Climático, el objetivo es estabilizar las concentraciones atmosféricas de gases de efecto invernadero; el Protocolo de Kioto tiene un doble objetivo: a) reducir las emisiones de estos gases en los países industrializados y $b$ ) promover los instrumentos de mercado que facilitan la reducción y absorción de emisiones de gases de efecto invernadero donde resulte económicamente más viable y eficiente (Fernández, 2010). En ese orden de ideas, Emerit Bono (2008) trata de contextualizar el cambio climático dentro del cambio global, apuntando hacia sus causas y consecuencias dentro sistema económico-social-medioambiental del siglo XXI. Asimismo, muestra que el cambio climático está inserto dentro de una perspectiva más amplia.

Por otro lado, Duarte (2006) precisa que el cambio global define al conjunto de transformaciones ambientales 
afectadas por la actividad humana, con especial referencia a las variaciones en los procesos que determinan el funcionamiento del sistema Tierra. Es decir, aquellas actividades que, aunque ejercidas localmente, tienen efectos que transcienden el ámbito local o regional para afectar el funcionamiento global del sistema.

De cualquier forma, el cambio es algo consustancial al planeta Tierra, que a lo largo de miles de millones de años de historia, ha experimentado cambios mucho más intensos que los que se avecinan. Como observa Duarte (2006), muchos de los cambios más importantes en la biósfera han estado forzados por organismos, como fue el paso de una biósfera pobre en oxígeno y con alta irradiación ultravioleta, a una biósfera con un $21 \%$ de oxígeno y una capa de ozono que filtra los rayos ultravioletas, consecuencia de la fotosíntesis en bacterias.

García (2007) muestra al cambio climático como la amenaza global más importante que enfrenta la humanidad en este siglo. Asegura, además, que es originado por la desmedida emisión de gases de efecto invernadero; estos están ocasionando el incremento de la temperatura promedio global y, con ello, la alteración del sistema climático, al igual que la elevación del nivel del mar.

García considera que este cambio, al alterar el régimen climático e hidrológico, ocasiona mayores sequías o lluvias más intensas con los consecuentes impactos sobre los procesos de desarrollo nacional y local. Por ejemplo, según el reporte de Global WaterPartnership (2005), los niveles de precipitación en África han bajado de 16 a 25\%, lo que ha acarreado una disminución de caudales de entre $50 \%$ y $70 \%$.

Destaca, además, desde su mirada particular, cómo los países en vías de desarrollo, como Perú (país latinoamericano), son los que menos han contribuido a la generación del problema, pero son los que más están sufriendo sus efectos, pues gran parte de sus recursos y medios de producción, son susceptibles al clima. Lo anterior, junto al reto y a la oportunidad que implica planear el desarrollo sostenible en un territorio complejo en términos territoriales y poblacionales, nos debe obligar a incluir en las políticas nacionales, consideraciones para la Reducción del Riesgo de Desastres (RRD) y la adaptación al cambio climático.

Si lo anterior no se lleva a cabo, seguramente aumentarán las cifras en cuanto a riesgos y desastres. $Y$ es que las pérdidas económicas globales por todo tipo de desastres se han incrementado de 200 billones de dólares en la década de 1970, a cerca de 750 billones en la década de 1990. Se estima que del total de desastres, al menos $74 \%$ son de origen climático. El Fenómeno El Niño (FEN) 1997-1998 generó 98 billones de dólares en pérdidas en todo el mundo (ISDR-UN, 2002).

De este análisis, se concluye que es ineludible un compromiso del Estado, la sociedad civil y el empresariado para alcanzar niveles de decisión política que permitan priorizar la prevención como una variable intrínseca para alcanzar el desarrollo. Con este fin, tiene suma importancia contar con una base científica que permita impulsar la formulación de políticas y la toma de decisiones. Mejorar las capacidades de respuesta de los gobiernos, regiones y comunidades es en muchos casos mejorar sus capacidades para hacerle frente al cambio climático (VARG, 2005).

\section{ADAPTACIÓN Y CAMBIO CLIMÁTICO}

El cambio climático ha obligado a la población a tomar medidas severas para mitigar y adaptarse a los diferentes sucesos que ocasiona este problema. Estudios realizados por el IPCC identifica cuatro ecosistemas o regiones particularmente vulnerables al cambio climático en el mundo.

El más significativo en términos físicos se encuentra en las regiones polares. Las temperaturas de los polos suben cerca del doble del promedio global. Ya estamos viendo los impactos del aumento de las temperaturas en el derretimiento del hielo polar tanto en la Antártida como en la región Antártica, así como en los glaciares en Los Andes y en otras montañas. A medida que la temperatura aumenta, el hielo se derrite y aumentan los niveles del mar. Si bien este es un fenómeno lento, es sin lugar a dudas muy real.

En cuanto a esto, deben tomarse medidas concretas para reducir un poco los riesgos que serán altos en los prolongados años si no existe atención seria e inmediata sobre estas medidas. Por tanto, es importante que se apliquen investigaciones a nivel local que brinden información para emplear medidas de mitigación y adaptación para estos sucesos; si bien existe una gran demanda por información sobre cómo enfrentar la adaptación al cambio climático, esta es todavía muy escasa. 
Por ejemplo, si revisamos los informes del IPCC, encontraremos gran cantidad de información a nivel global, que proporciona evidencia de lo que está sucediendo y de lo puede hacerse, pero no una buena cantidad a nivel local. Entonces, si uno se pregunta qué está sucediendo y qué debiera hacerse en Santiago de Chile, no se hallará mucha información en las publicaciones del IPCC que sea específica para las necesidades de esta ciudad.

Es de gran relevancia que se actué con planes que reduzcan los sucesos del cambio climático. Lo que necesitamos ahora es que todos los países en desarrollo comiencen a abordar la adaptación al cambio climático, que identifiquen cuáles son sus necesidades de adaptación y los distintos escenarios que enfrentan, para luego desarrollar estrategias. Y mientras avanzamos, debemos compartir el conocimiento y la experiencia que vamos adquiriendo con otros países, principalmente dentro de la región, pero también entre las regiones: América Latina, África y Asia, por ejemplo (Huq, 2007).

Levine (2007) declara que el cambio climático ha tomado un papel muy importante durante los últimos años. Latinoamérica, al igual que otras regiones con países en desarrollo, es altamente vulnerable a los efectos que ya está produciendo este problema ambiental. Sus limitaciones en mecanismos de respuesta y prevención la dejan expuesta a sufrir grandes impactos en sus comunidades causados por las transformaciones en las condiciones climáticas y la producción de eventos catastróficos.

Claramente, quienes más se resienten en situaciones de este tipo son las comunidades y las naciones más pobres. Es por esto que es necesario desarrollar planes de adaptación al cambio climático, el cual es inminente y con el que tendremos que aprender a convivir.

Se han plasmado medidas prácticas de adaptación al cambio climático a nivel local y global que puede ser implementado en cualquier país o comunidad; constan de tres pasos: (1) identificación y evaluación de los impactos y vulnerabilidad de los sistemas naturales y humanos, (2) construcción de capacidades y (3) medidas de adaptación. En Latinoamérica, algunos países han desarrollado avances en materia de adaptación ante efectos causados por el cambio climático, por ejemplo: Colombia inició un importante proyecto denominado «Piloto Nacional Integrado de Adaptación para Ecosistemas de Alta
Montaña, islas del Caribe Colombiano y Salud Humana (INAP)», cofinanciado por la cooperación bilateral (Holanda y Japón, entre otros). EI INAP trabaja en la formulación de un programa específico de adaptación para contrastar los efectos del cambio climático, con énfasis en los impactos sobre la salud humana (dengue y malaria). Es importante para la población latinoamericana que se empleen estas medidas, debido a que es favorable en todo sentido para el desarrollo de sus vidas.

Medidas innovadoras como la convocatoria a millones de personas de diferentes países a apagar las luces durante una hora, en lo que se conoce como la «Hora del Planeta» («EarthHour»), incentivan a la población global a actuar con una conciencia limpia ante el planeta y en contra al cambio climático global (Levine, 2007). Feo (2009)presenta una serie de evidencias de la realidad que nos afecta, tales como: (a) el aumento de la temperatura de la Tierra, donde apunta cómo durante el siglo XX, (b) la temperatura media de la superficie terrestre ascendió0,6 ㅇ aproximadamente y (c) unas dos terceras partes de este calentamiento se han producido desde 1975.

También se manifiestan en una diversidad de fenómenos meteorológicos que van desde el aumento de las corrientes eólicas (de aire), la alteración de los patrones del tiempo con sequías e inundaciones en muchas zonas, hasta la aparición de «El Niño» cada vez con mayor intensidad en los países de la costa del Pacífico (Trenberth, 1997).

Los climatólogos prevén que el calentamiento proseguirá a lo largo del siglo XXI junto con cambios en la pluviosidad y la variabilidad climática (McCarthy, 2001; MacCracken, 2008). A esto se le suma el deshielo de los polos y los glaciares, evidenciado en el marcado ritmo de fundición de los glaciares en las montañas del mundo, el cual presenta un aumento de 1,6 veces entre los años 2000 y 2005 en comparación con la década de 1990 y tres veces en relación con la década de 1980 (UNEP, 2008).

El consecuente aumento del nivel de los océanos y mares también es preocupante. Según Feo (2009), en el último siglo, el nivel del mar se ha elevado a una media de 1-2 $\mathrm{cm} /$ década y se prevé un aumento del nivel medio del mar de 28 a $43 \mathrm{~cm}$ para el año 2100.

Eso sin mencionar la frecuencia e intensidad de los fenómenos meteorológicos, pues los problemas del 
calentamiento global no sólo se manifiestan en el aumento de la temperatura del aire, en el deshielo de los glaciares y en el ascenso del nivel del mar; también se manifiestan en una diversidad de eventos que van desde el aumento de las corrientes eólicas (de aire), la alteración de los patrones del tiempo con sequías e inundaciones en muchas zonas, hasta la aparición de «El Niño» cada vez con mayor intensidad en los países de la costa del Pacífico (Trenberth, 1997).

A todo esto se le agrega: (a) la presencia de enfermedades hídricas, debido a que, según explica Feo (2009), los recursos hídricos se verán afectados en la medida en que los patrones de precipitación y evaporación cambien en todo el mundo, además, la propia escasez de agua puede forzar a las personas a utilizar recursos de agua dulce de menor calidad, a menudo contaminadas;(b) la presencia de enfermedades transmitidas por alimentos (ETA) ya sea por intoxicaciones o por infecciones, relacionadas con el deterioro de la calidad y disponibilidad de los alimentos, conjuntamente con la crisis del agua (Gregory, 2005); (c) la presencia de enfermedades respiratorias, donde se observa cómo la contaminación del aire y sus condiciones de temperatura y humedad repercuten en enfermedades, actuando también como determinantes del comportamiento de estas afectaciones (Shea, 2008);(d) la presencia de infecciones transmitidas por vectores, en las que se ven cómo las altas temperaturas alteran la distribución geográfica de las especies que transmiten enfermedades (Patz, 2006; Samalvides, 1999), lo que ocasiona un cambio en los patrones de transmisión y distribución estacional de muchas enfermedades (Cerda, 2008);(e) el cáncer y enfermedades crónico-degenerativas, producto de los contaminantes ambientales y las radiaciones solares intensas; $y$ ( $f$ ) la presencia de eventos de salud asociados con desastres naturales y temperaturas extremas, que hacen previsible el aumento de las enfermedades y muertes por estas causas, especialmente entre los más viejos y la población pobre de las ciudades (Basu, 2002; Fouillet, 2008).

Feo (2009), en su mismo artículo, define la mitigación como la acción sobre las causas; explica cómo la asociación saludambiente presupone una realidad compleja que exige un enfoque integrador de los aspectos biofísicos, socioeconómicos y político-culturales implicados (Rengifo, 2008).Esto debe estar complementado por la adaptación como la acción sobre las consecuencias, pues, según afirman Feo (2009), los efectos del calentamiento global ya han empezado a sentirse con mayor o menor intensidad en todas las regiones del mundo y se pronostica que se incrementen en frecuencia e intensidad. Es necesario preparar la respuesta para recuperarnos del mejor modo posible.

\section{CAMBIO CLIMÁTICO Y LOS SUELOS DE LATINOAMÉRICA}

Latinoamérica alberga alrededor del 40\% de las especies conocidas, además de ser la reserva más importante de agua y tierras cultivables del planeta. Sus bosques tropicales y templados representan cerca de un tercio de los bosques del mundo. Algunos sectores del bioma amazónico y del bosque templado subantártico se encuentran entre los menos intervenidos del mundo.

A pesar de toda esta riqueza biológica, importantes procesos de deforestación y desertificación están amenazando su patrimonio natural. De los 6.93 millones de $\mathrm{km} 2$ de bosques originales, en la actualidad, solo quedan 3.66 millones. Cada año se pierden 15.000 km2 de bosques, es decir, casi tres hectáreas por minuto .

Esto nos permite afirmar que aparte del cambio climático, el ser humano con sus actividades altera a gran escala los ecosistemas sin saber que los sistemas naturales tienen la capacidad para absorber pequeños desequilibrios o presiones generadas por factores ambientales cambiantes. Esta capacidad resiliente les permite a los ecosistemas permanecer inalterados frente a las oscilaciones climáticas o de otro orden. Así, la degradación de la tierra es consecuencia de una combinación de factores humanos y climáticos.

A través de la historia del planeta, las fluctuaciones climáticas han forzado importantes cambios en el paisaje . Es obvio que las actividades antrópicas de forma indirecta y directa contribuyen al deterioro de ecosistemas y al cambio climático. La degradación de la tierra es el resultado de la combinación de varias causas, tales como las prácticas agrícolas no adecuadas, la fragilidad de los ecosistemas, otras presiones de origen humano como contaminación y urbanización y las presiones naturales provenientes de un clima más variable y agresivo.

La degradación de la tierra es la primera fase de una cadena larga de los procesos que afectan la integridad de los 
ecosistemas, los servicios ambientales de estos y su capacidad para soportar las actividades humanas. Un ejemplo de esto es el fenómeno de El Niño y de La Niña. Durante la fase de El Niño, aguas del océano Pacífico se calientan 2 a $4{ }^{\circ} \mathrm{C}$ sobre lo normal, lo que acarrea precipitaciones intensas en el Cono Sur (Perú, Chile, Argentina); mientras que Colombia, Venezuela, México, NE de Brasil y la cuenca del Amazonas sufren de sequías.

Por otro lado, la fase fría es asociada con efectos inversos. Este fenómeno es una amenaza para los asentamientos humanos, es la causa principal de inundaciones y deslizamientos de tierras. Las sequías periódicas crean condiciones desfavorables para las inversiones en la agricultura. Esta oscilación oceánica es probablemente la mayor forzante de la variabilidad climática en el continente

Este ambiente de riesgo lleva a los agricultores a practicar una agricultura marginal, de bajo uso de insumos para reducir el riesgo económico. De este modo, la variabilidad climática es una causa primaria de pobreza y de las migraciones masivas de la población rural; este ha sido el caso del NE del Brasil, Argentina y Chile septentrionales y México. Actividades como esta deben ser reducidas concretamente para no seguir afectando los ecosistemas más de lo que está siendo afectado por el calentamiento global, causado también por el hombre.

\section{CAMBIO CLIMÁTICO EN LA AGRICULTURA DE COLOMBIA}

Si aterrizamos el tema de cambio climático en el contexto local (Colombia), podríamos dar el concepto que se tiene de este fenómeno frente a la agricultura del país y cómo está afectando los cultivos; de la misma forma, cuáles serían los impactos más relevantes sobre este sector.

Según estudios realizados por el Instituto de Hidrología, Meteorología y Estudios Ambientales (Ideam), Colombia contribuye con el $0,37 \%$ de las emisiones de GEI y es vulnerable al cambio climático (Ideam, 2010; Ideam, 2001). En el contexto agrícola, el territorio colombiano posee una gran capacidad para abastecer el mercado nacional y ha alcanzado una reconocida posición en productos de exportación. Sin embargo, el sector no solo debe enfrentar el reto que supone la baja productividad, uno de los más rezagados de la economía (DNP, 2008), sino que requiere implementar medidas efectivas de adaptación.
El cambio climático es una realidad (IPCC, 2007; Stern, 2006) y para la agricultura, se tiene el reto no solo de definir estrategias de adaptación, sino de implementarlas y monitorear sus resultados. Para tener una mayor concepción del cambio climático, únicamente no hay que analizar sus consecuencias, sino estudiar cuáles son las verdaderas causas que nos colocan en un estado de vulnerabilidad y saber qué decisiones tomar en el momento en que se presenten los desastres. Asimismo, evaluar el riesgo futuro para así poder estar preparados.

Existen evidencias científicas sobre el impacto del cambio climático en la agricultura por el efecto sobre las variables conexas relevantes como la temperatura, la precipitación, la concentración de dióxido de carbono y la humedad del suelo (IPCC, 2007). No obstante, las prácticas de gestión, los cambios tecnológicos, la dinámica del mercado y las políticas públicas afectan el sector y crean incertidumbres, debido a la dificultad de evaluar de forma aislada la influencia del clima con respecto a los otros factores (S.N., 2011).

Por tal motivo el IPCC (2007) reconoce la necesidad de profundizar en los estudios para demostrar las relaciones causa-efecto. Sin embargo, a pesar de las incertidumbres, también plantea impactos potenciales del cambio climático en la agricultura, sin contemplar medidas de adaptación (Fig. 1).

Algunas investigaciones (Adger, 2007; PNUD, 2007; PNUD, 2009) advierten que el cambio climático podría tener una incidencia directa sobre la productividad de las cosechas y sobre la fertilidad del suelo. Existe la probabilidad de variaciones en las tasas de degradación de la tierra, aumentos de salinización, incremento en las superficies de riesgo, mayores pérdidas de siniestros, reducciones de las especies polinizadoras y modificaciones importantes en la distribución y dinámica de las plagas y enfermedades. 


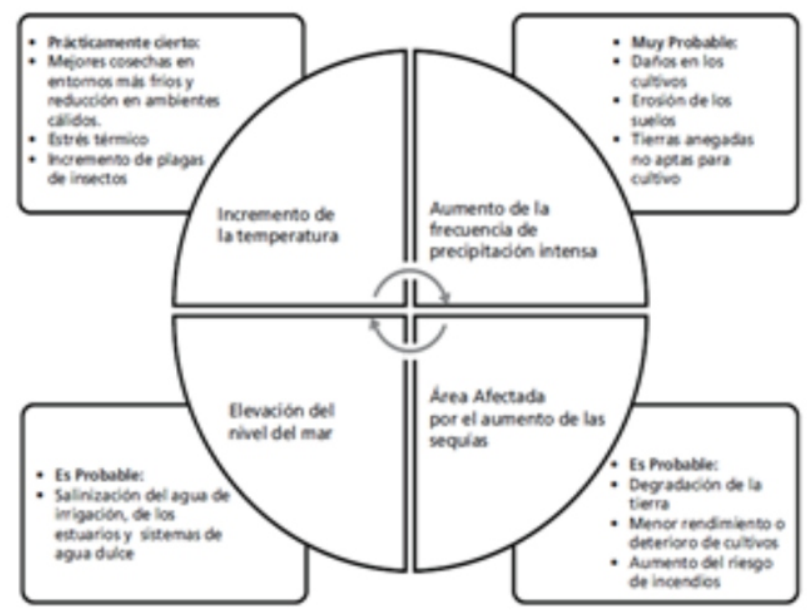

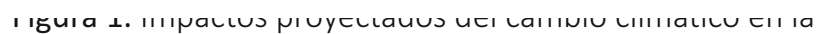
agricultura (IPCC, 2007).

Eduardo González y Cristian Hernández: Cambio climático: efectos sobre el suelo y la agricultura.

Un aspecto destacado en los estudios realizados por CEPAL (2009) y Perry (2004) es el efecto de fertilización por las elevadas concentraciones de $\mathrm{CO} 2$ en la atmósfera, que podría mitigar las repercusiones negativas del cambio climático en la productividad de los cultivos. No obstante, todavía existe incertidumbre sobre los posibles beneficios, porque hay muchas interacciones y factores de estrés que no pueden incorporarse a los modelos actuales (CEPAL, 2009; Ideam, 2007).

Para América Latina y el Caribe, la agricultura es una actividad económica que representa alrededor del 10\% del Producto Interno Bruto (PIB) y contribuye con el $12 \%$ de las exportaciones agrícolas mundiales. En la región, a partir de las estimaciones de la Organización de las Naciones Unidas para la Agricultura y la Alimentación (FAO) (2003), existen zonas con problemas de seguridad alimentaria, para las cuales los cultivos más sensibles han sido identificados: (a) Centroamérica y el Caribe: caña de azúcar, yuca, maíz, arroz y trigo; (b) Región Andina: palma, soya, caña de azúcar, yuca, papas, maíz, cebada, arroz y trigo; y (c) Brasil: soya, caña de azúcar, yuca, maíz, arroz y trigo.

Las investigaciones realizadas por el IPCC(2007; 2008) estiman aumentos en el rendimiento de algunos cultivos como la soya y la caña de azúcar y reducciones en el arroz, la cebada y la vid; mientras que para el trigo y el maíz, la respuesta es errática y depende del tipo de modelo.
Algunas cifras del Banco Mundial plantean que la productividad agrícola en Latinoamérica podría caer entre un $12 \%$ y un 50\% hacia el año 2100 (World Bank, 2009). Sin embargo, la vulnerabilidad varía según la región, los cultivos y las tecnologías aplicadas, también en función de la variabilidad climática natural por las modificaciones en los regímenes pluviales y de vientos y por la incidencia de fenómenos extremos (PNUD, 2000). Es importante anotar que la agricultura en pequeña escala es especialmente vulnerable y que los factores de estrés socioeconómicos suelen agravar dichas condiciones (IPCC, 2007).

En Colombia, las tierras con vocación agrícola corresponden al 18,9\% del territorio. EI PIB agropecuario ha contribuido con el $8 \%$ del PIB total y en promedio genera el 21\% de los empleos del país (Ideam, 2010; DNP, 2008). No obstante, el sector agrícola Colombiano es vulnerable tanto a la variabilidad climática natural como al cambio climático. Históricamente, la agricultura colombiana ha podido adaptarse a la variabilidad climática, pero los fenómenos extremos representan una gran amenaza para este sector (Banco de la República, 2007).

En Colombia, las emergencias y desastres guardan relación con las precipitaciones (Ideam, 2010; NOOA, 2011).Se ha detectado que los cambios en el régimen de lluvias tienen efectos muy diversos influenciados por El Niño-Oscilación del Sur (ENSO) (NOOA, 2011; ICA, 2010; Poveda, 2004). Los reportes asociados con sequía se elevan en un $21,6 \%$ durante los periodos de El Niño; mientras que en los años de La Niña, los desastres relacionados con la lluvia, como deslizamientos e inundaciones, aumentan en un $16,1 \%$ (Ideam, 2010).

Por ejemplo, la tragedia provocada por las graves inundaciones en el territorio Colombiano durante La Niña 2010-2011 llegó a tal magnitud, que desbordó las facultades ordinarias del Estado para atender la situación de desastre nacional por el deterioro y destrucción de la infraestructura vial y urbana, los derrumbes de diques y obras de contención, los daños en vías, las pérdidas de zonas agrícolas, de viviendas y el deterioro de los servicios públicos (Minhacienda, 2010).

Con respecto al cambio climático, la segunda Comunicación Nacional (Ideam, 2010), para el período comprendido entre el 2011 al 2040, estima niveles elevados de vulnerabilidad para el sector agropecuario y las 
áreas de terreno campesino, en especial, en las extensiones agrícolas de los departamentos de Cesar, Nariño, Cauca, Tolima, Magdalena, Córdoba y Antioquia. Los impactos potenciales altos podrían alcanzar el $50 \%$ de la superficie dedicada a las pasturas; el $57 \%$ de las áreas de cultivos permanentes y semipermanentes; el $71 \%$ del área sembrada de café y el $47 \%$ de los terrenos campesinos.

\section{CONCLUSIONES}

A manera de reflexión final, puede plantearse que independientemente de cuál sea la causa, ya sea por motivos antrópicos o naturales, o una mezcla entre las dos, el calentamiento global y el cambio climático es una realidad que debemos afrontar. Se debe asumir esta carga y plantear soluciones precisas y adecuadas para una correcta adaptación.

Queda claro que la mejor forma de adaptación al cambio climático va más allá, incluso, de la toma de decisiones. Transciende hacia la ejecución de planes y políticas justas, equitativas, viables $y$, por sobre todo, sostenibles de entendimiento o aceptación global con aplicación local, que mitiguen o reduzcan los impactos del cambio climático en el contexto particular y redunde en los mismos afectos, en mayor escala, sobre el contexto global.

Cabe destacar que de no realizarse ninguna acción, el cambio climático se convertirá en un óbice para el desarrollo económico y, mayoritariamente, para la productividad del sector agrícola, especialmente en países en desarrollo como Colombia, cuyos efectos más preponderantes auguran una pérdida sustancial de la vocación agrícola y del área cultivable del país.

\section{REFERENCIAS}

Adger, N (2007). «Assessment of adaptation practices, options, constraints and capacity». En Climate Change 2007: Impacts, Adaptation and Vulnerability. Londres: Cambrigde University Press.

Banco de la República (2007).El fenómeno del Niño y su posible impacto en Colombia. Bogotá. Ed. Banco de la Republica.

BasuR., SametJM (2002). "Relation between elevated ambient temperature and mortality: A review of the epidemiologic evidence». EpidemiolRev, 24(2), 190-202.

Bono,Emèrit (2008). Cambio climático y sustentabilidad económica y social: implicaciones sobre el bienestar social. Madrid: CIRIEC.

Comisión Económica para América Latina y el Caribe (Cepal) (2009). Cambio climático y desarrollo en América Latina y el Caribe: Una reseña. Santiago de Chile: Naciones Unidas.

Cerda,J., Valdivia,G.,Valenzuela,\&MT., Venegas,J. (2008). «Cambio climático y enfermedades infecciosas. Un nuevo escenario epidemiológico». Revista Chilena Infectol, 25(6), 447-52.

Departamento Nacional de Planeación (DNP) (2008). Documento CONPES 3527. Política Nacional de Competitividad y Productividad. Bogotá D.C.

Duarte, C. M. (Coord.) (2006).Cambio global. Impacto de la actividad humana sobre el planeta Tierra.CSIC. Ciudad: Madrid. ISBN: 978-84-00-08452-3

Feo, O., Solano, E., Beingolea, L., Aparicio, M., Villagra, M., Prieto, M. J., et al. (2009). «Cambio climático y salud en la región andina». Perú: MedExp Salud Pública, XXVI (1), 8393.

Fernández Cuesta, C.\&Fronti de García, L. (2010).«El cambio climático y su control económico en la empresa». Partida Doble, (226).

Fouillet, A., Rey, G., Wagner, V., Laaidi, K., Empereur Bissonnet, P., Le Tertre, A., et al. (2003) «Has the impact of 
heat waves on mortality changad in france since the European heat wave of summer 2003? A study of the 2006 heat wave». Int J Epidemiol, 37(2), 309-17.

Fred Singer, S. (2008). La Naturaleza, No la Actividad Humana, Controlan al Clima: Resumen para Hacedores de Políticas del Informe del Panel Internacional No Gubernamental Sobre Cambio Climático. Chicago, IL: The Heartland Institute.

García Vargas, J. (2007). "La necesidad de incorporar el cambio climático en las políticas de desarrollo». Caud. Difus., 12(23), 97-113.

Geisse, G. (2007). "Cambio climático y desarrollo sustentable». Ambiente y Desarrollo, 23(1)3-4.Santiago de Chile, 2007

Gregory, P.J., Ingram, J.S., \& Brklacich, M. (2005).Climate change and food security». PhilosTrans R SocLond B BiolSci, $360,2139-48$.

Grupo Intergubernamental de Expertos Sobre el Cambio Climático (IPCC) (2008). El Cambio Climático y el Agua. Bates, B.C., Z.W. Kundzewicz, S. Wu y J.P. Palutikof, Eds., 2008: El Cambio Climático y el Agua. Documento técnico del Grupo Intergubernamental de Expertos sobre el Cambio Climático, Secretaría del IPCC, Ginebra, 224 págs.

Huq, S. (2007). «Adaptación al cambio climático: Desafío para los próximos 20 años». Ambiente y Desarrollo, 23(1), 31-36.

Instituto Colombiano Agropecuario (ICA) (2010). Alertas Sanitarias y fitosanitarias en función de la predicción climática-recomendaciones de manejo fenómeno de La Niña.Ciudad: Bogotá.

Instituto de Hidrología, Meteorología y Estudios Ambientales (Ideam) (2001).Primera Comunicación Nacional ante la Convención Marco de las Naciones Unidas sobre el Cambio Climático. Bogotá D.C.: Trade Link Ltda.

Ideam (2010). 2a Comunicación Nacional ante la Convención Marco de las Naciones Unidas sobre el Cambio Climático. Bogotá D.C.: Trade Link Ltda.

International Strategy For Disaster Reduction (ISDR/ EIRD) (2002). Living withrisk. Ginebra, Suiza: Secretary.
Panel Intergubernamental sobre Cambio Climático (IPCC), (2007).Cambio climático 2007: Informe de síntesis. Contribución de los Grupos de trabajo I, II y III al Cuarto Informe de evaluación del Grupo Intergubernamental de Expertos sobre el Cambio Climático [Equipo de redacción principal: Pachauri, R.K. y Reisinger, A. (directores de la publicación)]. Ginebra, Suiza: IPCC.

IPCC (2008). Evaluación de la vulnerabilidad e impactos del cambio climático y del potencial de adaptación en América Latina. Lima, Perú: 1ra. Edición, 1ra. Impresión

IPCC. Cambio climático 2007: Informe de síntesis. Ginebra, Suiza: IPCC.

Levine, T. (2007). «Adaptación al cambio climático: Experiencia en América Latina». Ambiente y Desarrollo, 23(1), 41-47

Parry, M.L., Rosenzweig, C., Iglesias, A., Livermore, M., \&Fischer, G. (2004).Effects of climate change on global food production under SRES emissions and socio-economic scenarios», Global Environmental Change, 14(1).53-67.

MacCracken, M.C. (2008). «Prospects for future climate change and the reason for early action». J Air WasteManagAssoc., 58(6), 735-86.

Ministerio de Hacienda y Crédito Público (2010). Decreto 4831 de 2010. Bogotá.

NOOA (2011).Enso impacts on the US NOOA. National Weather Services. Climate Prediction Center. Recuperado de:http://www.cpc.ncep.noaa.gov/products/monitoring _ and_data/ENSO_connections.Shtml.

NRC, N. R. (2007). «Abrupt Climate Change».Agricultural and Forest Meteorology, 173, 234-254.

Patz, J.A., Olson, S.H. (2006). «Climate change and health: global to local influences on disease risk». Ann Trop Med Parasitol, 100(5-6), 535-49.

Programa de las Naciones Unidas para el Desarrollo (PNUD) (2000). Panorama del impacto ambiental de los recientes desastres naturales en América Latina y el Caribe. Lima, Perú: PNUD. 
PNUD (2007). Informe sobre Desarrollo Humano 2007/2008: La lucha contra el cambio climático: solidaridad frente a un mundo divido. Programa de las Naciones Unidad para el Desarrollo. Lima, Perú: Grupo Mundi Prensa. Poveda, G. (2004). «La hidroclimatología de Colombia. Una Síntesis desde la escala Interdecanal hasta la escala diurna». Rev. Acad. Colomb.Ciencia, 28 (107), 201-222.

Rengifo, H. (2008). "Conceptualización de la salud ambiental: teoría y práctica (parte 1)». Rev Peru Med Exp Salud Pública, 25(4), 403-9.

S.N. Seo. (2011). "An analysis of public adaptation to climate change using agricultural water schemes in South America». Ecological Economics. Vol 70, No 4, pp. 825-834. Samalvides, F., Núñez, R., Marquiño, W., Cabezas, C., \& Carrillo, C. (1999). "Cambio climático: evaluación de su impacto desde la perspectiva de la salud pública». En: Vulnerabilidad frente al cambio climático: aproximaciones a la experiencia con el fenómeno El Niño. Lima, Perú: Consejo Nacional del Ambiente.

Santibañez, F. (2007). «Cambio climático y degradación de tierras en Latinoamérica y chile». Ambiente y Desarrollo, 23(1), 54 .

Schnoor, J. (1996). Environmental modeling. Nueva York: A Willey-Interscience Series.

Shea, K.M., Truckner, R.T., Weber, R.W., \&Peden D.B. (2008).Climate change and allergic disease». J Allergy ClinImmunol, 122(3), 443-53.

Simona, T.F. (2006). Precipitation and temperature variability over south America from 1860 to 2100. Proceedings of 8 ICSHMO, 1527-1534.

Stern, N. The Economics of Climate Change. Londres: Cambridge UniversityPress, 2006.

Tierramérica (2009). América Latina ante los efectos irreversibles de un planeta más caliente. Ciudad: Montevideo. Programa de las Naciones Unidas para el Desarrollo (PNUD), Programa de las Naciones Unidas para el Medio Ambiente (PNUMA) y el Banco Mundial.

Trenberth, K.E. \&Hoar, T.J. (1997). «EI Niño and climatechange». Geophys Res Lett 24(23), 3057-60.
UNEP, WGMS (2008). Global glacier changes: facts and figures. Ginebra, Suiza: UNEP/WGMS.

Velázquez, F. (2008).25 preguntas sobre el cambio climático.Buenos Aires: Capital intelectual.

Vulnerability and Adaptation Resource Group (VARG) (2005).Disaster risk management in a changing climate. Kobe: The World Conference on Disaster Reduction, Outcomes Relevant to Climate Change Initiatives.

Wart, S. (2007). "Calentamiento Global». Ambiente y Desarrollo, 23(1), 81-82.

World Bank (2009). Respuestas Latinoamericanas al desafío del cambio climático. Washington, DC 20433. ISBN: 978-08213-7920-0 